\title{
Searches for very rare decays to purely leptonic final states at $\mathrm{LHCb}$
}

\section{Flavio Archilli*†}

Laboratori Nazionali di Frascati - INFN

E-mail: flavio.archillielnf.infn.it

Rare leptonic decays of the $B_{(s)}^{0}$ mesons are sensitive probes of New Physics. The LHCb experiment is well suited for these analyses due to its large acceptance and trigger efficiency, as well as its excellent invariant mass resolution and lepton identification capabilities. The latest $\mathrm{LHCb}$ results on the searches of $B_{(s)}^{0} \rightarrow \mu^{+} \mu^{-}$rare decay and $B_{(s)}^{0} \rightarrow e^{ \pm} \mu^{\mp}$ lepton flavour violating decay are presented.

The European Physical Society Conference on High Energy Physics -EPS-HEP2013 18-24 July 2013

Stockholm, Sweden

* Speaker.

${ }^{\dagger}$ on behalf of LHCb collaboration 


\section{Introduction}

To date, no effects of Physics beyond the Standard Model (SM) have been observed in the direct searches, but Nature provide many hints of the presence of totally new sector in addition to the well known particle "zoo". For example, the large asymmetry between matter and antimatter suggests a large CP-violating process carried by a new interaction. In the rare processes, where the SM prediction is very small, the effect of New Physics (NP) could arise at the same level, leading to an unambiguous interpretation of the results. Lepton Flavour Violating (LFV) decays, forbidden in the SM, could happen through tree level process mediated by a new particle not included in the SM. The study of these processes gives access to energy scales not probed by the direct searches. The rare decays, especially in the heavy flavour sector, are therefore the best place where to look at the effect of NP beyond the SM.

The $\mathrm{LHCb}$ detector is well suited to study B rare decays with muons in the final states. It benefits from a large acceptance for $b \bar{b}$ process in $p p$ collisions, a very efficient trigger for muons with low transverse momentum, a good momentum resolution, and a good muon identification efficiency. In this contribution, two of the latest searches of the rare decays with the LHCb experiment are presented: the evidence of the $B_{s}^{0} \rightarrow \mu^{+} \mu^{-}$decay and the search of the $B^{0} \rightarrow \mu^{+} \mu^{-}$decay, and the search of LFV decays $B_{(s)}^{0} \rightarrow e^{ \pm} \mu^{\mp}$.

2. $B_{(s)}^{0} \rightarrow \mu^{+} \mu^{-}$

The search of the rare decay $B_{(s)}^{0} \rightarrow \mu^{+} \mu^{-}$is one of the most promising ways to constrain the parameters of models with an extended Higgs sector. These decays are Flavour Changing Neutral Currents and helicity suppressed in the SM. The SM model predictions for these decays are [1]:

$$
\begin{aligned}
& \mathscr{B}\left(B_{s}^{0} \rightarrow \mu^{+} \mu^{-}\right)_{S M}=(3.2 \pm 0.2) \times 10^{-9} \\
& \mathscr{B}\left(B^{0} \rightarrow \mu^{+} \mu^{-}\right)_{S M}=(1.0 \pm 0.1) \times 10^{-10}
\end{aligned}
$$

In order to compare the experimental result and the theoretical prediction of the $\mathscr{B}\left(B_{s}^{0} \rightarrow \mu^{+} \mu^{-}\right)$, the experimental result has to be multiplied by $0.911 \pm 0.014$. This takes into account the effective lifetime of the $B_{s}^{0}$ meson.

The LHCb collaboration has reported the first evidence of the $B_{s}^{0} \rightarrow \mu^{+} \mu^{-}$decay with a $3.5 \sigma$ significance [2] in 2012 using $2 \mathrm{fb}^{-1}$ collected during the first two years of data taking. The result obtained was: $\mathscr{B}\left(B_{s}^{0} \rightarrow \mu^{+} \mu^{-}\right)=\left(3.2_{-1.2}^{+1.4}(\text { stat })_{-0.3}^{+0.5}(\right.$ syst $\left.)\right) \times 10^{-9}$. Using the same statistics the upper limit on the branching fraction of $B^{0} \rightarrow \mu^{+} \mu^{-}$is also obtained: $\mathscr{B}\left(B^{0} \rightarrow \mu^{+} \mu^{-}\right)<9.4 \times$ $10^{-10}$ at $95 \%$ ofC.L.. The combination of LHCb result with ATLAS and CMS set a more stringent upper limit on the branching fraction of $B^{0} \rightarrow \mu^{+} \mu^{-}$decay [3]: $\mathscr{B}\left(B^{0} \rightarrow \mu^{+} \mu^{-}\right)<8.4 \times 10^{-10}$ at 95\% ofC.L..

In this contribution the update of the previous analysis performed with the LHCb experiment is presented using the whole $3 \mathrm{fb}^{-1}$ dataset that includes the previously analysed dataset. In order to avoid any bias the mass region around the mass peaks of $B_{s}^{0}$ and $B^{0}$ is kept blind until the analysis completion. The structure of the analysis is the same used for the previous results but including important improvements in the discrimination and description of the background. The signal and 
background classification is done in the di-muon invariant mass $m_{\mu \mu}$ and the multivariate classifier output plane. The multivariate classifier is a boosted decision tree, BDT, which is improved with respect to the previous analysis. The classifier is trained using kinematic and geometrical variables. The training is performed on samples of simulated signal and background events and calibrated on data. The signal yield is converted into a branching fraction by normalizing to two control channels, $B^{0} \rightarrow K^{+} \pi^{-}$and $B^{+} \rightarrow J / \psi K^{+}$. Once the analysis is finalized, the events in the signal region are compared with the expectation. An unbinned likelihood fit to the data is performed to evaluate the branching fraction of $B_{s}^{0} \rightarrow \mu^{+} \mu^{-}$. The upper limit on the $\mathscr{B}\left(B^{0} \rightarrow \mu^{+} \mu^{-}\right)$is assessed using the CLs method [4].

$B_{(s)}^{0} \rightarrow \mu^{+} \mu^{-}$candidates are selected as two oppositely charged tracks, with a transverse momentum $p_{T}$ in the range $[0.25,40] \mathrm{GeV} / c$ and a momentum $p<500 \mathrm{GeV} / c$. The two tracks have to form a secondary vertex displaced from any $p p$ interaction vertex. After the selection, the surviving backgrounds are mainly due to random combinations of muons generated in B semileptonic decays $\left(b \bar{b} \rightarrow \mu^{+} \mu^{-} X\right)$ and peaking background from $B_{(s)}^{0} \rightarrow h^{+} h^{\prime}-$ where hadrons are misidentified as muons. A first multivariate classifier based on kinematic and geometrical variables is trained to be effective on the combinatorial background. The peaking background is reduced using the particle identification variables. Other background sources due to the misidentification of one or two candidate muons are taken into account and included in the final result. The calibration of the BDT expected output is based on a data-driven procedure. The BDT signal distribution is obtained by fitting a sample of inclusive $B_{(s)}^{0} \rightarrow h^{+} h^{\prime}$ - decays. These distributions are corrected for the biases due to the trigger and muon identification. Since the BDT classifier is trained using also the decay time of the mother particle, an additional correction to the signal PDF is applied in order to take into account the discrepancy between the decay time distributions of the signal and control channel used for the calibration. The correction is evaluated assuming, for the $B_{s}^{0}$, a SM decay time distribution. For the combinatorial background $b \bar{b} \rightarrow \mu^{+} \mu^{-} X$, the BDT shape is obtained by interpolating in each BDT bin the mass sidebands into the signal region with an exponential PDF. The invariant dimuon mass PDF's for the signals are described by a Crystal Ball function where the mean values are calibrated using the exclusive $B_{(s)}^{0} \rightarrow h^{+} h^{\prime}-$ decays, while the resolutions are evaluated from the di-muon resonances and exclusive $B_{(s)}^{0} \rightarrow h^{+} h^{\prime-}$. The combinatorial background mass shape is obtained by fitting the data sidebands with an exponential function. The peaking background is assumed to have the same BDT distribution of the signal while the mass shape is evaluated from simulated events. The number of candidates $N_{B_{(s)}^{0} \rightarrow \mu^{+} \mu^{-}}$observed in the signal window are converted into a branching fraction by using the following equation:

$$
\mathscr{B}\left(B_{(s)}^{0} \rightarrow \mu^{+} \mu^{-}\right)=\frac{\mathscr{B}_{\text {norm }} \varepsilon_{\text {norm }} f_{\text {norm }}}{N_{\text {norm }} \varepsilon_{\text {sig }} f_{\text {sig }}} \times N_{B_{(s)}^{0} \rightarrow \mu^{+} \mu^{-}}
$$

where $N_{\text {norm }}$ and $\mathscr{B}_{\text {norm }}$ are the number and the branching fraction of normalization events respectively. The efficiency $\varepsilon_{\text {sig(norm) }}$ for signal (normalization) channel is the product of the reconstruction efficiency, the selection efficiency and the trigger efficiency. The control channels used are $B^{+} \rightarrow J / \psi K^{+}$and $B^{0} \rightarrow K^{+} \pi^{-}$. The term $f_{\text {norm }} / f_{\text {sig }}$ is the fraction of the probability for a $b$ quark to fragment into the corresponding $B_{q}$ meson. $f_{d}$ and $f_{u}$ are assumed to be equal. The $f_{s} / f_{d}$ is measured by $\mathrm{LHCb}$ using the data recorded at $\sqrt{s}=7 \mathrm{TeV}$ in hadronic decays, $B_{s}^{0} \rightarrow D_{s}^{-} \pi^{+}$ and $B^{0} \rightarrow D^{-} K^{+}$, and in semileptonic decays, $B_{s}^{0} \rightarrow D_{s} \mu X$ and $B^{0} \rightarrow D^{+} \mu^{-} X$. The result is 
$f_{s} / f_{d}=0.259 \pm 0.015$ [5] [6]. The stability of $f_{s} / f_{d}$ as a function of $\sqrt{s}$ is verified by comparing the ratios of yield of $B_{s}^{0} \rightarrow J / \psi \phi$ and $B^{+} \rightarrow J / \psi K^{+}$. The effect of the measured dependence of $f_{s} / f_{d}$ on $p_{T}$ is found to be negligible. Assuming $B_{(s)}^{0} \rightarrow \mu^{+} \mu^{-}$to be SM the selected data sample $40 \pm 4 B_{s}^{0} \rightarrow \mu^{+} \mu^{-}$and $4.5 \pm 0.4 B^{0} \rightarrow \mu^{+} \mu^{-}$are expected in the full BDT range and with a mass in $[4900,6000] \mathrm{MeV} / c^{2}$.

The value of $\mathscr{B}\left(B_{s}^{0} \rightarrow \mu^{+} \mu^{-}\right)$and $\mathscr{B}\left(B^{0} \rightarrow \mu^{+} \mu^{-}\right)$are evaluated from a simultaneous unbinned maximum-likelihood fit on data performed in projection of $m_{\mu \mu}$ in each BDT bin. The yields of combinatorial background, $B_{s}^{0} \rightarrow \mu^{+} \mu^{-}$and $B^{0} \rightarrow \mu^{+} \mu^{-}$are left free, while the yields and the shapes of exclusive backgrounds are constrained to their expectations. An excess of $B_{s}^{0} \rightarrow \mu^{+} \mu^{-}$ candidates over the background only hypothesis is observed with a statistical significance of $4 \sigma$. The statistical significance for the $B^{0} \rightarrow \mu^{+} \mu^{-}$process is of $2 \sigma$. The result of the simultaneous fit is [7]:

$$
\begin{aligned}
& \mathscr{B}\left(B_{s}^{0} \rightarrow \mu^{+} \mu^{-}\right)=\left(2.9_{-1.0}^{+1.1}(\mathrm{stat})_{-0.1}^{+0.3}(\mathrm{syst})\right) \times 10^{-9}, \\
& \mathscr{B}\left(B^{0} \rightarrow \mu^{+} \mu^{-}\right)=\left(3.7_{-2.1}^{+2.4}(\mathrm{stat})_{-0.4}^{+0.6}(\mathrm{syst})\right) \times 10^{-10}
\end{aligned}
$$

In Fig. 1 the dimuon mass distribution is shown for the events with a BDT $>0.7$. Since no significant excess is observed of $B^{0} \rightarrow \mu^{+} \mu^{-}$decays, a modified frequentist approach $\mathrm{CL}_{s}$ is used to assess an upper limit on the branching fraction. The $\mathrm{CL}_{s}$ distribution as a function of $B^{0} \rightarrow \mu^{+} \mu^{-}$branching fraction is reported in Fig. 1. The branching fraction on $B^{0} \rightarrow \mu^{+} \mu^{-}$is constrained to be less than $6.3(7.4)$ at $90 \%(95 \%)$ of C.L..
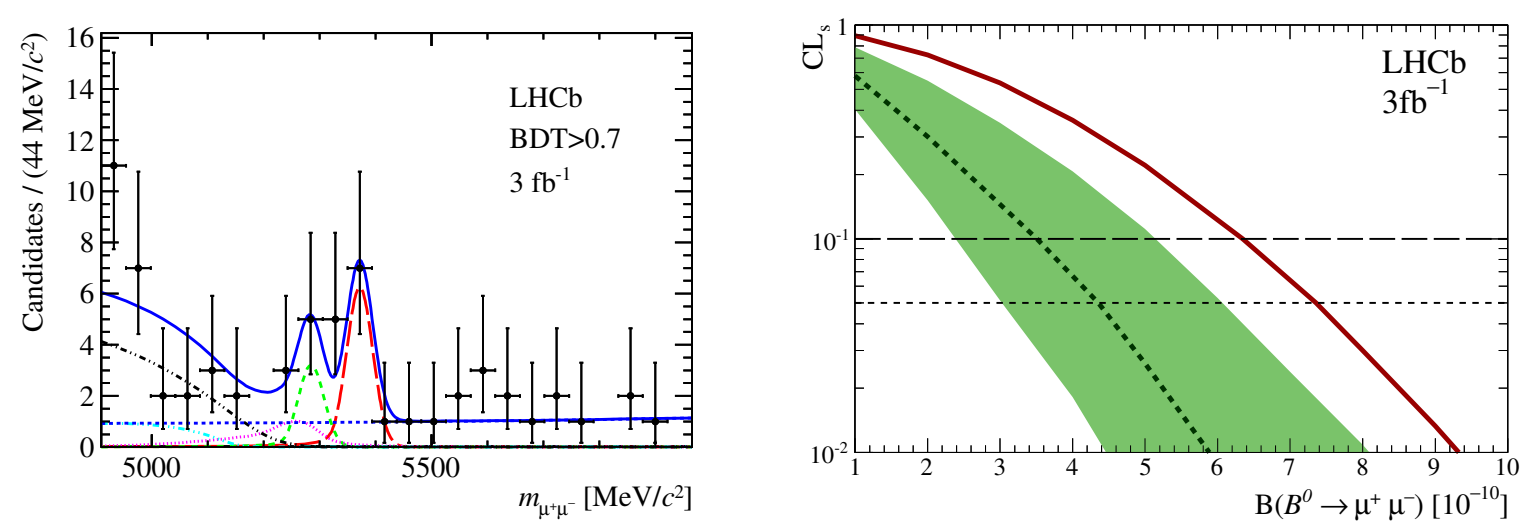

Figure 1: On the left plot the invariant mass distribution of the $B_{(s)}^{0} \rightarrow \mu^{+} \mu^{-}$selected events with BDT>0.7 (black dots) is shown together with the result of the fit (blue solid line) and each component: $B_{s}^{0} \rightarrow \mu^{+} \mu^{-}$ (red dashed line), $B^{0} \rightarrow \mu^{+} \mu^{-}$(green dashed line), $B_{(s)}^{0} \rightarrow h^{+} h^{\prime}$ (magenta dotted line), $B^{0(+)} \rightarrow \pi^{0(+)} \mu^{+} \mu^{-}$ (light blue dot-dashed line), $B^{0} \rightarrow \pi^{-} \mu^{+} v$ and $B_{s}^{0} \rightarrow K^{-} \mu^{+} v$ (black dot-dashed line). On the right plot $\mathrm{CL}_{s}$ as a function of the branching fraction of the $B^{0} \rightarrow \mu^{+} \mu^{-}$. The dashed black line represents the expected $\mathrm{CL}_{s}$ in the background-only hypothesis. The green area represents the $1 \sigma$ region. The solid red line is the observed $\mathrm{CL}_{s}$.

3. $B_{(s)}^{0} \rightarrow e^{ \pm} \mu^{\mp}$

Charged LFV decays, like $B_{(s)}^{0} \rightarrow e^{ \pm} \mu^{\mp}$ have never been observed before and they are forbid- 
den in the SM up to $\mathscr{O}\left(10^{-54}\right)$. Many models with a local gauge symmetry between leptons and quarks, like the Pati-Salam model [8], allow these processes. In this model the new interaction between lepton and quarks is mediated by spin 1 gauge bosons, called Pati-Salam leptoquarks (LQ) that carry color and lepton quantum numbers. The previous best upper limits on the branching fractions were obtained by the CDF Collaboration and are $\mathscr{B}\left(B_{s}^{0} \rightarrow e^{ \pm} \mu^{\mp}\right)<2.0(2.6) \times 10^{-7}$ and $\mathscr{B}\left(B^{0} \rightarrow e^{ \pm} \mu^{\mp}\right)<6.4(7.9) \times 10^{-8}$ [9]. These results correspond to lower limits on the value of the masses of the leptoquarks of $M_{\mathrm{LQ}}\left(B_{s}^{0} \rightarrow e^{ \pm} \mu^{\mp}\right)>47.8(44.9) \mathrm{TeV} / c^{2}$ and $M_{\mathrm{LQ}}\left(B^{0} \rightarrow e^{ \pm} \mu^{\mp}\right)>$ $59.3(56.3) \mathrm{TeV} / c^{2}$ at $90 \%$ (95\%) of C.L. [9].

The analysis presented in this contribution is based on $1 \mathrm{fb}^{-1}$ collected by the LHCb experiment in 2011 at $\sqrt{7} \mathrm{TeV}$. In order to avoid any bias, the signal mass range is kept blind until the completion of the analysis. This analysis inherited the general structure from the strategy used for the search for the $B_{(s)}^{0} \rightarrow \mu^{+} \mu^{-}$decay. The topological and kinematic informations are exploited to reduce most of the background. The number of observed events are then compared with the expected number of signal and background events in the plane of invariant mass, $m_{e \mu}$, and the output of the Boosted Decision Tree, BDT. The main background component is due to random combination of electron-muon pairs produced by the semileptonic decays of to different $b$-hadrons. The other sources of background are due to $\Lambda_{b}^{0} \rightarrow p l^{-} \bar{v}_{l}, B_{c}^{+} \rightarrow J / \psi\left(e^{+} e^{-}\right) \mu^{+} v_{\mu}, B_{c}^{+} \rightarrow J / \psi\left(\mu^{+} \mu^{-}\right) e^{+} v_{e}$ and the peaking background $B_{(s)}^{0} \rightarrow h^{+} h^{-} \rightarrow e^{+} \mu^{-}$. The effect of these processes have been taken into account in the final result. The invariant mass line shape of the $B_{(s)}^{0} \rightarrow e^{ \pm} \mu^{\mp}$ decays is described by a double Crystal Ball function where the parameters are obtained from a sample of simulated events. The BDT distribution for the signal is determined from data sample using $B_{(s)}^{0} \rightarrow h^{+} h^{\prime}$ control channel. The BDT output and the invariant mass shape for the background in the signal mass region is evaluated by interpolating with an exponential function the mass distribution of data sidebands in bins of BDT output. The number of expected signal events is then obtained by normalizing to the $B^{0} \rightarrow K^{+} \pi^{-}$channel. The compatibility of the observed events in the $m_{e \mu}$ and BDT plane with the expected distribution for a given branching fraction hypothesis is evaluated using the CLs method [4]. The expected and the observed CLs as a function of the
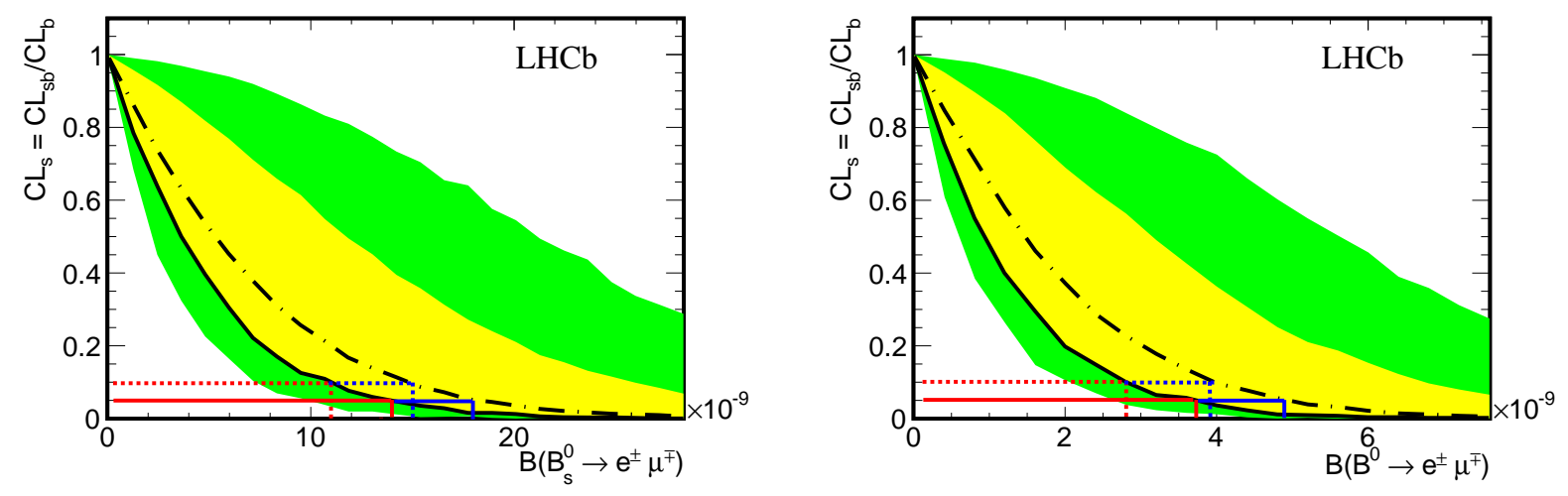

Figure 2: $\mathrm{CL}_{s}$ as a function of the branching fraction for $B_{s}^{0} \rightarrow e^{ \pm} \mu^{\mp}$ (left) and $B^{0} \rightarrow e^{ \pm} \mu^{\mp}$ (right) decays. The dashed lines are the expected $\mathrm{CL}_{s}$ in the background-only hypothesis. The yellow (green) area represents the $1 \sigma(2 \sigma)$ region of the expected $\mathrm{CL}_{s}$ distribution. The solid lines represents the observed $\mathrm{CL}_{s}$. The upper limits at $90 \%$ and $95 \%$ are indicated by a dotted and a solid blue lines respectively for the expectation and by red lines for the observation. 
branching fraction are shown in Fig. 2 for the $B_{s}^{0} \rightarrow e^{ \pm} \mu^{\mp}$ and $B^{0} \rightarrow e^{ \pm} \mu^{\mp}$ channels. This analysis set the limits on $\mathscr{B}\left(B_{s}^{0} \rightarrow e^{ \pm} \mu^{\mp}\right)<1.1(1.4) \times 10^{-8}$ and $\mathscr{B}\left(B^{0} \rightarrow e^{ \pm} \mu^{\mp}\right)<2.8(3.7) \times 10^{-9}$ at $90 \%$ $(95 \%)$ of C.L. [10].

The branching fraction of the LFV process is related, in the Pati-Salam framework, to the mass of the lepto-quark as shown in Fig. 3. Using the upper limits on the branching fractions just obtained the following bounds on $M_{\mathrm{LQ}}$ are found: $M_{\mathrm{LQ}}\left(B_{s}^{0} \rightarrow e^{ \pm} \mu^{\mp}\right)>107(101) \mathrm{TeV} / c^{2}$ and $M_{\mathrm{LQ}}\left(B^{0} \rightarrow e^{ \pm} \mu^{\mp}\right)>135(126) \mathrm{TeV} / c^{2}$ at $90 \%$ (95\%) of C.L. [10].
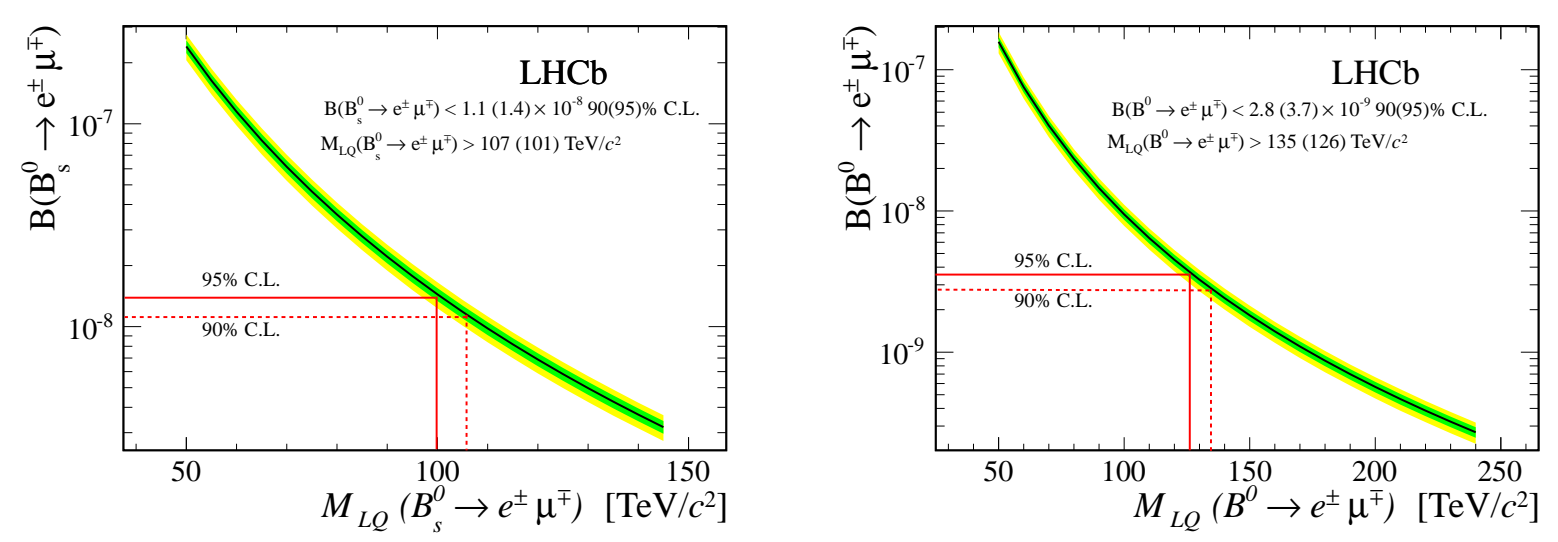

Figure 3: Branching fraction as a function of the mass of the leptoquark for the $B_{s}^{0} \rightarrow e^{ \pm} \mu^{\mp}$ (left) and $B^{0} \rightarrow e^{ \pm} \mu^{\mp}$ (right). The green (yellow) area represents the $1 \sigma(2 \sigma)$ region.

\section{Conclusions}

Rare B decays represent a powerful probe to search for new physics effects beyond the SM. The LHCb experiment is demonstrating its power in search for rare decays in the Heavy Flavour sector. In this proceeding a new result for $B_{(s)}^{0} \rightarrow \mu^{+} \mu^{-}$decay is presented using the sample of $3 \mathrm{fb}^{-1}$ collected in 2011 and 2012. This analysis confirmed the evidence for the $B_{s}^{0} \rightarrow \mu^{+} \mu^{-}$with a significance of $4.0 \sigma$ and put a new world best limit on the $B^{0} \rightarrow \mu^{+} \mu^{-}$branching fraction. The LHCb collaboration obtained also the new world best limit on the $B_{(s)}^{0} \rightarrow e^{ \pm} \mu^{\mp}$ decay, improving the previous result by a factor of $\sim 20$. Moreover the new bounds on the lepto-quark mass in the Pati-Salam framework are a factor 2 higher than the previous limits.

\section{References}

[1] A. J. Buras, J. Girrbach, D. Guadagnoli and G. Isidori, Eur. Phys. J. C72 (2012) 2172, arXiv:1208.0934.

[2] R. Aaij et al. [LHCb Collaboration], Phys. Rev. Lett. 110, 021801 (2013), arXiv:1211.2674.

[3] LHCb Collaboration, LHCb-CONF-2012-017.

[4] A. Read, J. Phys. G28 (2002) 2693.

[5] R. Aaij et al. [LHCb Collaboration], JHEP 1304 (2013) 001, arXiv:1301.5286.

[6] LHCb Collaboration, LHCb-CONF-2013-011. 
[7] R. Aaij et al. [LHCb Collaboration], Phys. Rev. Lett. 111 (2013) 101805, arXiv:1307.5024.

[8] J. C. Pati and A. Salam, Phys. Rev. D 10, 275 (1974).

[9] T. Aaltonen et al. (CDF Collaboration), Phys. Rev. Lett. 102, 201801 (2009).

[10] R. Aaij et al. [LHCb Collaboration], Phys. Rev. Lett. 111 (2013) 141801, arXiv:1307.4889. 